\begin{tabular}{|c|l|}
\hline Title & Potential-dependent structure of the interfacial water on the gold electrode \\
\hline Author(s) & $\begin{array}{l}\text { Nihonyanagia, Satoshi; Y ea, Shen; U osaki, Kohei; Dreesen, Laurrent; Humbert, Christphe; Thiry, Paul; Peremans, } \\
\text { Andre }\end{array}$ \\
\hline Citation & $\begin{array}{l}\text { Surface Science, 573(1), 11-16 } \\
\text { https://doi.org/10.1016j.Susc.2004.04.059 }\end{array}$ \\
\hline Issue Date & 2004.12-01 \\
\hline Doc URL & http://hdl.handle.net/2115/20569 \\
\hline Type & article (author version) \\
\hline File Information & ss573.pdf \\
\hline
\end{tabular}

Instructions for use 


\section{Potential Dependent Structure of the Interfacial Water on the Gold Electrode}

Satoshi Nihonyanagi, Shen $\mathrm{Ye}^{+}$, and K. Uosaki*

Physical Chemistry Laboratory, Division of Chemistry, Graduate School of Science, Hokkaido University, Sapporo 060-0810, Japan

Laurrent Dreesen, Christphe Humbert, Paul Thiry, and Andre Peremans

Laboratoire de Spectroscopie Moleculaire de Surface, Department of Physics, Facultes Universitaires Notre-Dame de la Paix, B-5000 Namur, Belgium

Corresponding author:

Kohei Uosaki,

Physical Chemistry Laboratory,

Division of Chemistry, Graduate School of Science,

Hokkaido University,

Sapporo 060-0810, JAPAN

Tel: +81-11-706-3812

Fax: +81-11-706-3440

E-mail: uosaki@PCL.sci.hokudai.ac.jp

+ Present address: Catalysis Research Center, Hokkaido University. 


\begin{abstract}
Doubly-tunable Sum-Frequency Generation (SFG) spectra demonstrate that the water molecules at gold/electrolyte interface change their orientation with applied potential. At negative potentials, water molecules in the double layer align with their oxygen atom pointing to the solution. As potential became positive to be close to the potential of zero charge (pzc), the SFG signal decreased, suggesting the $\mathrm{OH}$ groups of the water molecule are either in random orientation or parallel to the electrode. As potential became more positive than the pzc, the SFG signal increased again with the oxygen-up orientation same as in the negative potential region, indicating that water molecules interact with the adsorbed sulfate anions. The peak position of the SFG spectra indicates a relatively disordered state of water molecules at the gold electrode surface, in contrast to the previously observed ice-like structure of water at electrolyte/oxide interfaces.
\end{abstract}

Keywords: sum frequency generation, electrochemical methods, surface structure, water, metal-electrolyte interfaces. 
Interfacial water molecules play prominent roles in many physical, chemical and biological processes. Although many theoretical studies on the structure of water molecules at various interfaces are available, most of the experimental investigations on the water structure have been carried out in the vapor or bulk aqueous phase [1-3] due to the lack of an effective experimental probe for interfacial study. The structure of the electrode/solution interface under potential control is one of the most important issues in electrochemistry [4] and many spectroscopic techniques such as surface enhanced Raman spectroscopy (SERS)[5, 6], surface infrared spectroscopy[7], surface enhanced infrared spectroscopy (SEIRAS)[8, 9] and X-ray diffraction [10] have been used to investigate the water structure at the electrode/electrolyte interface during the last two decades. X-ray diffraction study, for example, suggested that the water molecules at a silver electrode in $\mathrm{NaF}$ solution flipped their dipole when the electrode potential was changed from negative to positive[10].

Vibrational spectroscopy enables not only to determine the orientation of water dipole but also to identify the hydrogen-bonding state of water, i.e., order of water structure. Recently, Sum frequency generation (SFG) spectroscopy [11-13] has emerged as a powerful vibrational spectroscopy to investigate the structures of water molecules at buried interfaces. As SFG is a second order non-linear optical process, which is forbidden in the bulk of a centro-symmetric electrolyte and electrode, the analysis depth of SFG is intrinsically limited to the layer of molecules that are aligned at the interface, in contrast to IR spectroscopy in which signal from bulk water dominates. Furthermore, SFG is free from the ambiguity associated to the choice of reference spectrum and can avoid the necessity of working with a rough electrode as required for SEIRAS and SERS. Actually, SFG has successfully been applied to investigate the structure of the water at liquid/gas and liquid/solid interfaces[14-17]. However, the strong absorption of the IR by both the metal electrode and the bulk water makes the detection of the interfacial water at a smooth electrode difficult[18]. Although SFG in internal reflection configuration with ultrathin metal film [19, 20] can avoid the IR absorption, the existence of the overwhelming nonresonant signal from the gold film still prevents the detection of the weak signal from the interfacial water. 
In the present study, we employed a doubly tunable laser system [21-23] for the SFG measurement in internal reflection at the gold electrode/electrolyte interface so that the strong nonresonant contribution from the gold can considerably be suppressed and the resonant signal from the water molecules can be emphasized. At negative potentials, the water molecules in the double layer align with their oxygen atom pointing to the solution. As potential became positive to be close to the potential of zero charge (pzc), the $\mathrm{OH}$ groups of the water molecules are either in random orientation or parallel to the electrode. As potential became more positive than the pzc, the SFG signal increased again. Quantitative analysis of the SFG spectra, which takes into account the phase angle between the resonant and nonresonant contributions, suggests that the water molecules at the positive potential takes the oxygen-up orientation as same as in the negative potential region. The oxygen-up orientation at both negative and positive potential regions contrasts with the simple flip-flop behavior of the water molecules reported previously[10, 24], indicating the water molecules interact with the adsorbed sulfate anions at the positive potentials.

The gold films of $25 \mathrm{~nm}$ thick were evaporated on IR-grade fused quartz plates with a $5 \mathrm{~nm}$ titanium buffer layer in a vacuum chamber of $2 \times 10^{-6}$ Torr. The deposition rate and substrate temperature were kept at less than $0.01 \mathrm{~nm} / \mathrm{s}$ and $200{ }^{\circ} \mathrm{C}$, respectively, in order to obtain a flat film. A Pt foil and Ag/AgCl electrode were used as a counter and reference electrodes, respectively. All the potentials in this paper are quoted against $\mathrm{Ag} / \mathrm{AgCl}$. A cyclic voltammogram of the gold film demonstrated that the surface of the gold film was dominated by wide (111) domains.

The doubly tunable laser system [21, 22] consists of two OPO's synchronously pumped by a mode-locked Nd:YAG laser $(25 \mathrm{~Hz})$. The two OPOs built around a $\mathrm{LiNbO}_{3}$ and a $\mathrm{BBO}$ crystal generate 9 ps pulses tunable from 2.5 to $4 \mu \mathrm{m}$ (bandwidth: $2 \mathrm{~cm}^{-1}$ ) and from 460 to $700 \mathrm{~nm}$ (bandwidth: $3 \mathrm{~cm}^{-1}$ ) with output powers of $10 \mathrm{~mW}$ and $30 \mathrm{~mW}$, respectively. The incident angles of the quasi co-linear visible and infrared beams onto the cell window are $55^{\circ}$ and $65^{\circ}$, respectively. About $5 \%$ of the incident IR and visible beam power is used to generate a reference SFG signal in a 
ZnS polycrystalline to compensate for any fluctuation of the infrared beam power. The polarizations of the SFG, visible and IR beams are s, s and p, respectively.

Since the strong nonresonant contribution from the gold due to the gold d-s inter-band transition centered at $550 \mathrm{~nm}$ is the reason for a relatively low signal/noise ratio in the SFG spectra measured at $532 \mathrm{~nm}$ excitation, the suppression of the nonresonant contribution and the emphasis of the resonant contribution can be expected when the visible wavelength is tuned outside the inter-band transition[25]. The optimum wavelength for the SFG measurements of the gold surface was determined by examining the SFG spectra of the ODT monolayer adsorbed on a 30-nm thick gold film using the internal reflection geometry with various visible wavelengths in the region between 470 and $700 \mathrm{~nm}$. The resonance to non-resonance ratio for the peak at $2880 \mathrm{~cm}^{-1}$ attributed to the $\mathrm{CH}_{3}$ symmetric stretching was lowest when $532 \mathrm{~nm}$ light was used. The highest ratio was obtained for the visible wavelength of $470 \mathrm{~nm}$ as about 0.5 , which was considered to be high enough to resolve the $\mathrm{OH}$ bands. Thus, we employed the visible wavelength of $470 \mathrm{~nm}$ to observe the $\mathrm{OH}$ bands of the water on the gold electrode.

Figure 1(a)-(d) shows SFG spectra in the $\mathrm{OH}$ stretching region obtained at the gold electrode in $10 \mathrm{mM}$ sulfuric acid solution at various electrode potentials using the visible wavelength of $470 \mathrm{~nm}$. A broad band centered at around $3500 \mathrm{~cm}^{-1}$ was observed with a shoulder around $3250 \mathrm{~cm}^{-1}$. The signal in the $3000-3100 \mathrm{~cm}^{-1}$ region was rather constant and seemed to show the level of the non-resonant background. The SFG intensity was the lowest at $+0.6 \mathrm{~V}$ and the highest at $-0.2 \mathrm{~V}$.

To confirm that the broad band around $3500 \mathrm{~cm}^{-1}$ is really due to the $\mathrm{OH}$ vibration of water molecules, the experiment was repeated by replacing $\mathrm{H}_{2} \mathrm{O}$ by $\mathrm{D}_{2} \mathrm{O}(99.8 \%$ ) in the electrolyte solution. The SFG spectra obtained in the $\mathrm{D}_{2} \mathrm{O}$ solution (Fig. 1e) showed neither a clear peak nor clear potential dependence. The fact that the SFG spectra in the $\mathrm{H}_{2} \mathrm{O}$ solution showed a large potential dependence while that in the $\mathrm{D}_{2} \mathrm{O}$ solution showed no peak and did not change with potential clearly indicates that the broad band observed in the spectra is attributed to the $\mathrm{OH}$ stretching of the interfacial water molecules. Although an investigation in the OD vibration region 
should be ideal to confirm the potential dependent water structure, the limited transparency range of the fused quartz plate prevents the detection of the OD bands near $4 \mu \mathrm{m}$.

The experimental data were fitted to the following equation [24, 26] assuming two peaks around $3500 \mathrm{~cm}^{-1}$ and $3250 \mathrm{~cm}^{-1}$, which are known to be of "liquid-like water", representing the asymmetric $\mathrm{OH}$ stretching $\left(v_{3}\right)$ of the asymmetrically hydrogen bonded water molecules in a disordered arrangement and of “ice-like water", representing the symmetric OH stretching $\left(v_{1}\right)$ of tetrahedrally coordinated water molecules, respectively[24, 27].

$$
I_{S F G}=\left|\sum_{n} \frac{A_{n}}{\omega_{I R}-\omega_{n}+i \Gamma}+\right| \chi_{N R}^{(2)}\left|e^{i \varepsilon}\right|^{2}
$$

where $\omega_{\mathrm{IR}}$ is the frequency of the incident IR light and $\chi_{\mathrm{NR}}, \varepsilon, \mathrm{A}_{\mathrm{n}}$ and $\Gamma_{\mathrm{n}}$ are the vibrationally-nonresonant component, the phase angle between the resonant and nonresonant contributions, the strength and damping constant of the surface vibration or rotation mode with frequency $\omega_{\mathrm{n}}$, respectively.

The strengths of the peak at $3500 \mathrm{~cm}^{-1}\left(\mathrm{~A}_{3500}\right)$ were plotted against the applied potential in Figure 2. The intensity ratio between the two peaks at $3500 \mathrm{~cm}^{-1}$ and $3250 \mathrm{~cm}^{-1}$ can be considered as an indicator of the order of the interfacial water[24].

The spectrum obtained at $-0.2 \mathrm{~V}$ (Fig. 1a) resembled the SEIRA spectra reported by Ataka et al. $[8,9]$. The intensity of the peak at $3500 \mathrm{~cm}^{-1}$ was higher than that of $3250 \mathrm{~cm}^{-1}$ in contrast to the previously reported results observed at electrolyte/oxides interface where the peak at $3250 \mathrm{~cm}^{-1}$ corresponding to the ice-like structure of water was higher than that of $3250 \mathrm{~cm}^{-1}[24,26]$. This result indicates that the interfacial water molecules at the negatively charged gold electrode are rather weakly hydrogen bonded, i.e., disordered. This is also in good agreement with the molecular dynamics simulation for electric double layers, which suggests the lack of hydrogen bonding of the water molecules in the first layer on the metal electrode[3]. The relatively large SFG intensity at the negative potential region suggests that layers of water molecules align rather perpendicular to the surface with their oxygen atom pointing to the solution.

The SFG intensity was the lowest at $+0.6 \mathrm{~V}$ (Fig. 1c), suggesting either the structure of the 
interfacial water molecules was more random or the direction of the dipole orientation was closer to parallel to the surface, i.e., water molecules were less SFG active, at this potential than those at other potentials. The SEIRAS study [8,9] as well as molecular simulation [28] also suggested that the dipole of the interfacial water molecules was parallel to the surface at around the pzc, which is reported to be about $0.3 \mathrm{~V}$ in a solution containing $0.05 \mathrm{M} \mathrm{KClO}_{4}, 0.02 \mathrm{M} \mathrm{HClO}_{4}$ and $5 \mathrm{mM}$ $\mathrm{K}_{2} \mathrm{SO}_{4}[29]$.

As potential became more positive, the SFG intensity increased further, showing water molecules align rather perpendicular to the surface again. It is noteworthy that SFG spectrum gives direct information for the absolute orientation of molecules at an interface, which cannot be obtained by IR or Raman spectroscopy. If the direction of the dipole of interfacial water is changed, the phase angle between the resonant and nonresonant contributions, $\varepsilon$, should be also changed by $\pi$. Thus, the upward SFG peak should become a downward dip if the interfacial water molecules flip when the potential was changed from negative to positive across the pzc because the flipping of interfacial water molecules gives the inverse phase relative to the nonresonant contribution from the gold[24, 26, 30, 31]. It is clearly demonstrated in Fig. 3 where experimental result obtained at -0.2 $\mathrm{V}$ with a fitting curve ( $\varepsilon=100$ deg.), and the simulated SFG spectrum using the same parameters except for the $\varepsilon$, which is changed by $\pi$ ( $\varepsilon=-80$ deg.) are shown. The fact that the relative phase did not change in all the potential regions as shown in Fig.1 (a)-(d) indicates that the interfacial water molecules did not flip within this potential region and interfacial water molecules still point their oxygen atom to the solution even at potentials more positive than the pzc. This is in contrast to the previous report by Toney et al. They showed that the position of the oxygen atom of the water molecule was different for the negative and positive potentials at the Ag (111) electrode surface in $\mathrm{NaF}$ solution, where the specific adsorption of $\mathrm{Na}^{+}$and $\mathrm{F}^{-}$ions are negligible, based on an X-ray scattering and suggested that the interfacial water molecules flipped[10].

The present behavior can be explained by taking the existence of an adlayer of the sulfate anions into account. It is well known that sulfate anions are adsorbed on a gold electrode at potentials more 
positive than $0.2 \mathrm{~V}$ and form an ordered adlayer at $0.9 \mathrm{~V}[29,32,33]$. Shi et al. showed the adsorbed species from a solution containing $0.05 \mathrm{M} \mathrm{KClO}_{4}, 0.02 \mathrm{M} \mathrm{HClO}_{4}$ and $5 \mathrm{mM} \mathrm{K}_{2} \mathrm{SO}_{4}$ is sulfate, $\mathrm{SO}_{4}{ }^{2-}$, not bisulfate, $\mathrm{HSO}_{4}^{-}$, and net charge of the sulfate adlayer/electrode is negative based on the chronocoulmetric measurement[29]. Thus, the water molecules orient with their hydrogen atoms pointing to the surface, i.e., oxygen-up orientation in the positive potential region due to the interaction between water molecules and adsorbed sulfate anions. Actually, recent electrochemical scanning tunneling microscopy (EC-STM)[34] and SEIRAS[9] investigations suggested that water molecules are incorporated into the ordered adlayer of sulfate anions at the positively charged noble metal electrode in a sulfuric acid solution, although these technique cannot provide the information of the absolute orientation of the water molecules. The present results demonstrate that the orientation of water molecules at the electrode depends not on the charge of the electrode itself but on the net charge and the structure of the adlayer/electrode.

In conclusion, the potential dependent SFG spectra of the interfacial water at the gold electrode/electrolyte solution interface were obtained for the first time by employing $470 \mathrm{~nm}$ as the visible input light. The experiment with heavy water confirmed that the bands observed in the $\mathrm{OH}$ stretching region came from the interfacial water molecules. The orientation of water molecules at the electrode was found to depend not on the charge of the electrode itself but on the net charge and the structure of the adlayer/electrode. Potential dependent orientation change of water molecules are schematically shown in Fig. 4. At potentials more negative than the pzc, the water molecules in the vicinity of the electrode align on the surface with their hydrogen atoms pointing to the surface (Fig. 4(a)). Interfacial water molecules oriented randomly or more likely with their hydrogen atoms pointing nearly parallel to the surface around the pzc (Fig. 4(b)). At potentials more positive than the pzc, water molecules are incorporated with sulfate anions with their hydrogen atoms pointing to the surface as is the case in the negative potential region (Fig. 4(c)).

Further investigations including the effects of the electrolyte, its concentration, and the surface modifications on the potential dependent behavior of the interfacial water molecules are now in progress. 


\section{Acknowledgment.}

This work was partially supported by Grants-in-Aid for Scientific Research (No. 13304047) from the Ministry of Education, Culture, Sports Science and Technology, Japan and FONDS National de la Recherche Scientifique, Belgium. SN acknowledges the Japan Society for the Promotion of Science for a Fellowship for Young Scientist. Mr. Nishida is acknowledged for his help in the preparation of the gold films. 


\section{References}

[1] D. Eisenberg and W. Kauzmann, The Structure and Properties of Water (Oxford University Press, London, 1969).

[2] P. A. Thiel and T. E. Madey, Surf. Sci. Rep. 7 (1987) 211.

[3] J. Lipkowski and P. N. Ross, Structure of Electrified Interfaces (VCH Publishers, Inc., New York, 1993).

[4] J. O. M. Bockris and A. K. N. Reddy, Modern Electrochemistry 2A, 2nd Edition: Fundamentals of Electrodics. (Plenum, New York, 2001).

[5] M. Fleischmann, P. J. Hendra, I. R. Hill and M. E. Pemble, J. Electroanal. Chem. 117 (1981) 243.

[6] B. Pettinger, M. R. Philpott and J. G. Gordon II, Surf. Sci. 150 (1981) 415.

[7] F. Kitamura, T. Ohsaka and K. Tokuda, J.Electroanal. Chem. 412 (1996) 183.

[8] K. Ataka, T. Yotsuyanagi and M. Osawa, J. Phys. Chem. B 100 (1996) 10664.

[9] K. Ataka and M. Osawa, Langmuir 14 (1998) 951.

[10] M. F. Toney, J. N. Howard, J. Richer, G. L. Borges, J. G. Gordon, O. R. Melroy, D. G. Wiesler, D. Yee and L. B. Sorensen, Nature 368 (1994) 444.

[11] Y. R. Shen, The Principles of Nonlinear Optics (John Wiley \& Sons, Inc., New York, 1984).

[12] A. Tadjeddine and A. Peremans, in Spectroscopy for Surface Science, edited by R. J. H. Clark and R. E. Hester (John Wiley \& Sons Ltd, Chichester, UK, 1998), p. 159.

[13] C. D. Bain, J. Chem. Soc. Faraday Trans. 91 (1995) 1281.

[14] P. B. Miranda and Y. R. Shen, J. Phys. Chem. B 103 (1999) 3292.

[15] D. E. Gragson and G. L. Richmond, J. Phys. Chem. B 102 (1998) 3847.

[16] C. Schnitzer, S. Baldelli and M. J. Shultz, J. Phys. Chem. B 104 (2000) 585.

[17] Q. Du, R. Superfine, E. Freysz and Y. R. Shen, Phys. Rev. Lett. 70 (1993) 2313.

[18] F. Dederichs, in Institut fur Grenzflachenforchung and Vakuumphysik (Forschungszentrum Julich, Julich, 1999).

[19] C. T. Williams, Y. Yang and C. D. Bain, Lagmuir 16 (2000) 2343. 
[20] S. Ye, S. Nihonyanagi, K. Fujishima and K. Uosaki, in Studies in Surface Science and Catalysis, edited by Y. Iwasawa (Elsevier Science, 2001), Vol. 132, p. 705.

[21] A. A. Mani, L. Dressen, C. Humbert, P. Hollander, Y. Caudano, P. A. Thiry and A. Peremans, Surf. Sci. 502-503 (2002) 261.

[22] A. A. Mani, L. Dressen, P. Hollander, C. Humbert, Y. Caudano, P. A. Thiry and A. Peremans, Appl. Phys. Lett. 79 (2001) 1945.

[23] M. B. Raschke, M. Hayashi, S. H. Lin and Y. R. Shen, Chem. Phys. Lett. 359 (2002) 367.

[24] Q. Du, E. Freysz and Y. R. Shen, Phys. Rev. Lett. 72 (1994) 238.

[25] A. L. Rille and A. Tadjeddine, J. Electroanal. Chem. 467 (1999) 238.

[26] S. Ye, S. Nihonyanagi and K. Uosaki, PCCP 3 (2001) 3463.

[27] J. R. Scherer, in Advances in Infrared and Raman Spectroscopy, edited by R. J. H. Clark and R. E. Hester (Heyden, Philadelphia, 1978), Vol. 5.

[28] G. Nagy and K. Heinzinger, J. Electroanal. Chem. 327 (1992) 25.

[29] Z. Shi, J. Lipkowski, M. Gamboa, P. Zelenay and A. Wiekowski, J. Electroanal. Chem. 366 (1994) 317.

[30] M. S. Yeganeh, S. M. Dougal and H. S. Pink, Phys. Rev. Lett. 83 (1999) 1179.

[31] O. Pluchery and A. Tadjeddine, J. Electroanal. Chem. 500 (2001) 379.

[32] O. M. Magnussen, J. Hangbock, J. Hotlos and R. J. Behm, Faraday Discuss. 94 (1992) 329.

[33] G. J. Edens, X. Gao and M. J. Weaver, J. Electranal. Chem. 375 (1994) 357.

[34] L.-J. Wan, S.-L. Yau and K. Itaya, J. Phys. Chem. 99 (1995) 9507. 


\section{Figure captions}

Figure 1. SFG spectra in the $\mathrm{OH}$ stretching region obtained at the gold electrode in $10 \mathrm{mM}$ sulfuric acid solution using visible wavelength of $470 \mathrm{~nm}$ at (a) $-0.2 \mathrm{~V}$, (b) $+0.2 \mathrm{~V}$, (c) $+0.6 \mathrm{~V}$ and (d) +1.0 $\mathrm{V}$ and fitted curves (solid lines). (e) SFG spectra in the $\mathrm{OH}$ stretching region obtained at the gold electrode in $10 \mathrm{mM}$ sulfuric acid $\mathrm{D}_{2} \mathrm{O}(99.8 \%)$ solution at $-0.2 \mathrm{~V}$ : open circle and $+0.9 \mathrm{~V}$ : filled circle. Electrolyte solutions were purged with ultra-pure argon gas prior to each experiment.

Figure 2. The strengths of the peak at $3500 \mathrm{~cm}^{-1}\left(\mathrm{~A}_{3500}\right)$ of the spectra shown in Fig. 1(a)-(d) as a function of the applied potential.

Figure 3. SFG spectrum shown in Fig. 1 (a) with the fitted curve (solid line) to Eq. (1) using $\varepsilon=100$ deg and a simulated SFG spectrum with the same parameters of the fitted curve except for $\varepsilon=-80$ deg (dotted line).

Figure 4. Schematic models of the interfacial structure at potentials (a) more negative than, (b) around and (c) more positive than the pzc. Arrows show the direction of the water dipole. 
Figures

Figure 1 Nihonyanagi et al.

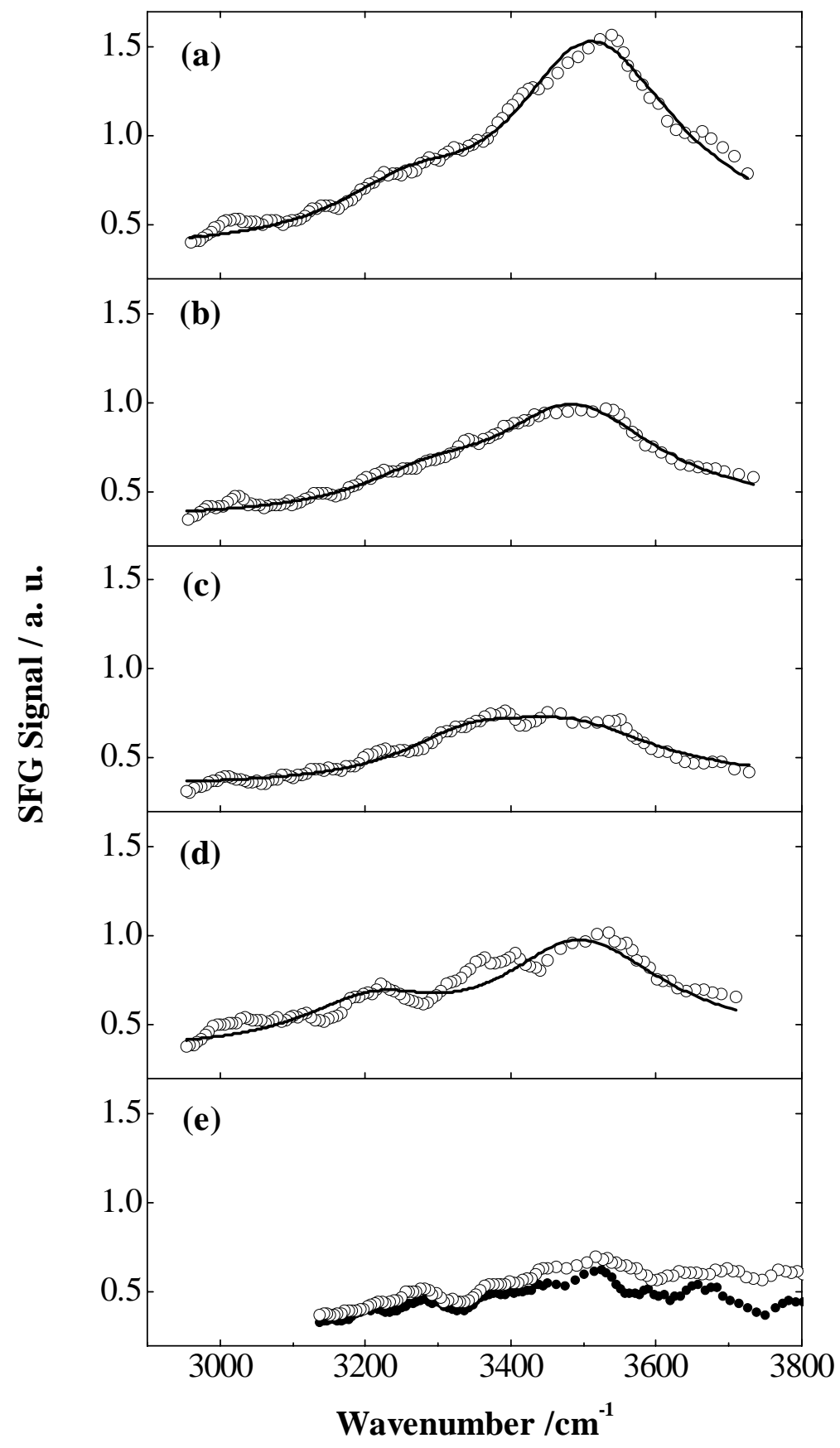




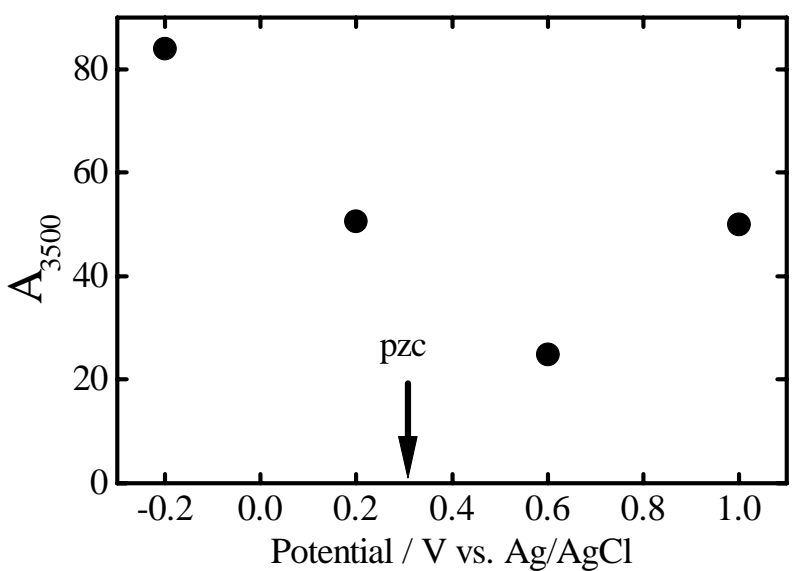

Figure 3 Nihonyanagi et al.

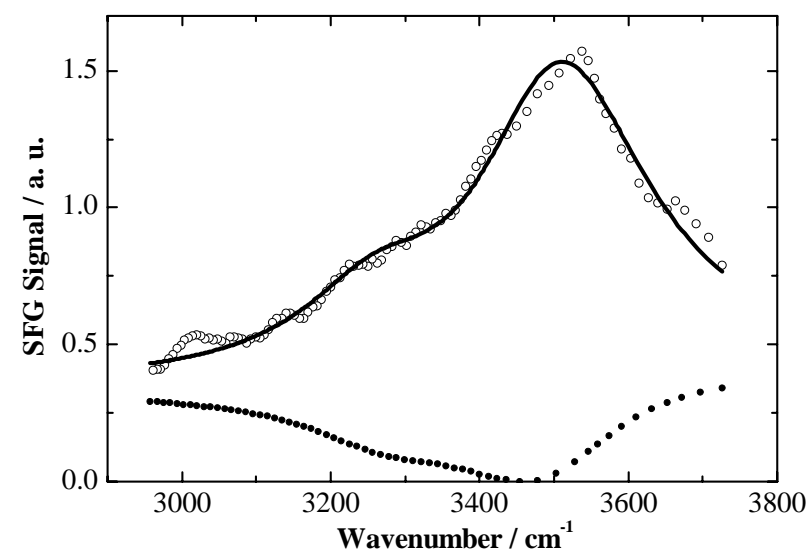

Fig. 4 Nihonyanagi et al.

(a)

(b)

(c)

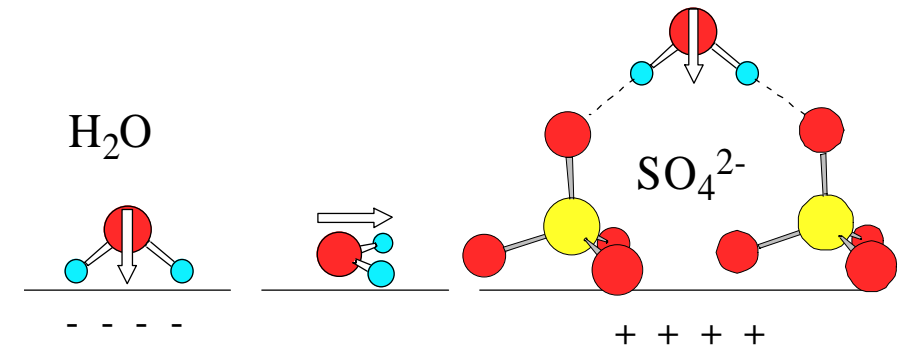

